\title{
Rational chemical multifunctionalization of graphene interface enhances targeting cancer therapy
}

Matteo Andrea Lucherelli, ${ }^{\#}$ Yue Yu, ${ }^{\ddagger}$ Giacomo Reina, ${ }^{\#}$ Gonzalo Abellán, ${ }^{\dagger}$ Eijiro Miyako, ${ }^{\ddagger *}$ Alberto Bianco, $\#$,*

${ }^{\#}$ CNRS, Immunology, Immunopathology and Therapeutic Chemistry, UPR 3572, University of Strasbourg, ISIS, 67000 Strasbourg, France

Department of Materials and Chemistry, Nanomaterials Research Institute (NMRI), National Institute of Advanced Industrial Science and Technology (AIST), Tsukuba, Ibaraki, 305-8565, Japan

$\dagger$ Instituto de Ciencia Molecular (ICMol), Universidad de Valencia, Catedrático José Beltrán 2, 46980, Paterna, Valencia, Spain

Corresponding author: a.bianco@ibmc-cnrs.unistra.fr, e-miyako@jaist.ac.jp

Keywords: Carbon materials; graphite; multifunctionalization; diazonium salts; cancer therapy 


\begin{abstract}
The synthesis of a drug delivery platform based on graphene was achieved through a step-bystep strategy of selective amine deprotection and functionalization. The multifunctional graphene platform, functionalized with indocyanine green, folic acid and doxorubicin showed an enhanced anticancer activity. The remarkable targeting capacity for cancer cells in combination with the synergistic effect of drug release and photothermal properties prove the great advantage of a combined chemo- and phototherapy based on graphene against cancer, opening the doors to future therapeutic applications of this type of material.
\end{abstract}


In the last decades, the interest on the applications of nanomaterials in nanomedicine has raised up very fast. New drug delivery platforms, based on different types of nanoparticles, carbon nanotubes, graphene, and other materials, have been developed. ${ }^{[1,2]}$ The large variety of reactions offered by the chemistry of these materials and their intrinsic properties permit the combination features, like specific cell targeting, controlled drug release, photothermal and photodynamic therapies, and imaging. ${ }^{[3-5]}$ Drugs, as doxorubicin or paclitaxel, present great anticancer activity but low water solubility that reduce their therapeutic efficacy and suffers of undesired side effects. ${ }^{[6]}$ A great advantage of nanomaterials as drug delivery systems consists on their ability to complex high quantity of hydrophobic drugs on their surface, improving the uptake and release of the drug in cancer cells, and reducing the non-selectivity of the free drugs. ${ }^{[2,7]}$ Moreover, fundamental properties to consider in the development of new systems are their biocompatibility, the amount of drug loading, the interactions with the biological environment and the biodistribution into the body after administration. ${ }^{\left[{ }^{[8}\right.}$ Within the wide range of nanomaterials, graphene has been found to have a good biocompatibility and biodegradability, leading to explore this form of carbon as drug delivery carrier. ${ }^{[9,10]}$ Moreover, its high surface/mass ratio (theoretical: $2630 \mathrm{~m}^{2} / \mathrm{g}$ ) allows a high drug loading, by absorbing organic molecules through $\pi-\pi$ stacking or hydrophobic interaction. Thanks to an easy functionalization and to its good water dispersibility, graphene oxide (GO), the oxidized form of graphene, has been largely studied as drug carrier. Indeed, following the pioneer work of Dai and co-workers, ${ }^{[11]}$ many other groups have investigated the applications of GO for drug delivery. ${ }^{[1]} \mathrm{GO}$ and its derivatives, thanks to their easy process of functionalization, have been widely employed to produce drug delivery platform. ${ }^{[12]}$ On the other hand, this easy functionalization can lead to a non-selective site reaction and, eventually, side reactions with amines present in the organism. Moreover, the highly defective GO surface is able to form radical species and the material is not stable in reducing conditions, with the risk to modification of the functional groups, the stability and the chemical properties. Graphene is presenting a higher chemical stability respect to GO to reductive conditions and fewer interactions with cells membrane due to functional groups on its surface. However, to the best of our knowledge no published studies have reported the use of pristine graphene as drug delivery carrier, despite its great potential in nanomedicine. ${ }^{[13]}$ Following the past studies performed by our group on the multifunctionalization of carbon nanotubes, ${ }^{[3]}$ we have designed and synthetized a covalent multifunctional graphene platform (mfG), suitable for cancer therapy applications. Our study has focused on targeting, tracking, delivery and photothermal activity of mfG on cancer cells. We selected indocyanine green (ICG) as fluorophore to follow the uptake and to track the 
material inside the cells. Folic acid (FA) was covalently bound through a PEG linked to graphene, to specifically target the cancer cells, and doxorubicin (Dox) was used as anticancer drug. We investigated the in vitro properties of this multifunctional conjugate using HeLa cells, showing very promising results and low toxicity, as confirmed by in vivo biocompatibility tests performed on mice.

The platform designed for our study is characterized by the presence of three functionalities, ICG as fluorophore, FA as targeting agent and DOX as therapeutic molecule. The starting material to prepare this platform corresponds to a multifunctional graphene recently described (Figure S1). ${ }^{[14]} \mathrm{mfG}$, obtained through one-pot covalent multifunctionalization of $\mathrm{KC}_{8}$ with diazonium salts, is bearing three orthogonally protected benzylamine derivatives that allow a specific strategy of step-by-step amine deprotection and functionalization. Following the sequential removal of each protecting group, the order of introduction of the new functionalities is crucial for achieving the final desired conjugate. The chemical stability of each functional group under the deprotection conditions of each amine was initially investigated, in order to establish the synthetic route. The optimized strategy, shown in Scheme 1 (see SI for complete synthetic details), started from phthalimide (Pht) deprotection (1) followed by the amidation with 4-pentynoic acid (2), introducing an alkyne as first functional group. The selective reactivity of the alkyne with azides and its stability to tertbutyloxycarbonyl (Boc) and benzyloxycarbonyl (Cbz) deprotection conditions make this functional group an orthogonal reactive site respect to the other protected amines. The common characterizations techniques for organic reactions, such as TLC and ${ }^{1} \mathrm{H}-\mathrm{NMR}$ are not applicable to follow the functionalization of graphene nanomaterials, making the characterization of each reaction step difficult and sometime uncertain. To understand the evolution of the reaction, we exploited the presence of primary amines, applying the colorimetric Kaiser test, to evaluate the amount of free amines before and after the reactions ${ }^{[15]}$ The quantity of primary amines after step (1) resulted $55 \mu \mathrm{mol} / \mathrm{g}$, and decreased to $14 \mu \mathrm{mol} / \mathrm{g}$ after the amidation (2), corresponding to a final loading of 4-pentynoic acid of $\sim 41 \mu \mathrm{mol} / \mathrm{g}$ (75\% yield). Through this procedure we were able to easily evaluate the loading of each subsequent functionality on $\mathrm{mfG}$. 


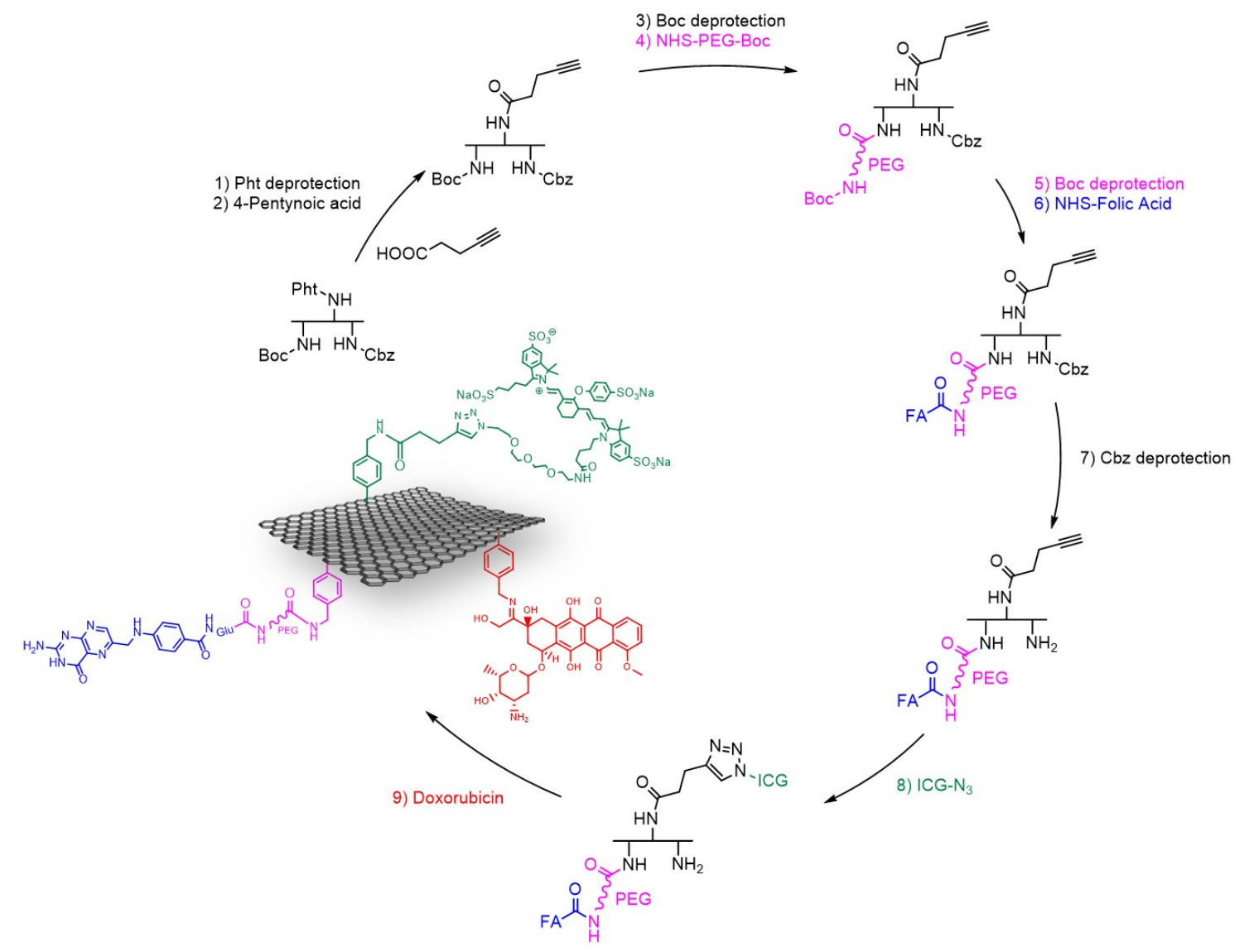

Scheme 1: Schematic representation of the synthetic routes followed to the functionalize $\mathrm{mfG}$ to obtain mfG/PEG-FA/ICG/Dox.

After the introduction of the alkyne moiety, Boc protection was removed (3) $(\sim 33 \mu \mathrm{mol} / \mathrm{g})$ and the amine was functionalized with a PEG chain protected with a Boc at the amino group and activated as N-hydroxysuccinimide (NHS) at the carboxylic function (NHS-PEG-Boc) (MW, $3000 \mathrm{Da})$ (4) (zero $\mu \mathrm{mol} / \mathrm{g}$ of free amines was measured, quantitative yield). The PEG chain plays two important roles: to increase water dispersibility and the biocompatibility of graphene and to act as a spacer for the linking of FA to the conjugate. After Boc deprotection of the PEG chain (5) $(\sim 30 \mu \mathrm{mol} / \mathrm{g})$, the amines were functionalized with a preformed NHS-FA derivative (6) (zero free amines were measured after the coupling, quantitative yield). FA stability to $\mathrm{Cbz}$ deprotection was already demonstrated by Ménard-Moyon et al. on carbon nanotubes allowing the next steps of chemical modification. ${ }^{[3]}$ The removal of $\mathrm{Cbz}$ group under strong acid conditions (7) liberated the last amines ( $\sim 30 \mu \mathrm{mol} / \mathrm{g}$ free amine), employed for the final step of $\mathrm{mfG}$ functionalization. Before this reaction, indocyanine green was introduced by click chemistry (8). In this case the reaction was monitored by fluorescence measurement of functionalized graphene in NMP. Due to partial quenching of the fluorescence by graphene, ${ }^{[16]}$ it is not possible to quantitative evaluate the loading of ICG through direct spectroscopic 
comparison. To prove that the observed fluorescence was due to the covalent grafting of the fluorophore and not to ICG physisorbed onto the surface, a control reaction was performed in absence of the click copper catalyst. No fluorescence was measured on this control reaction while the conjugate obtained from step (8) had a maximum of emission at $818 \mathrm{~nm}$, confirming the covalent reaction (Figure 1a).

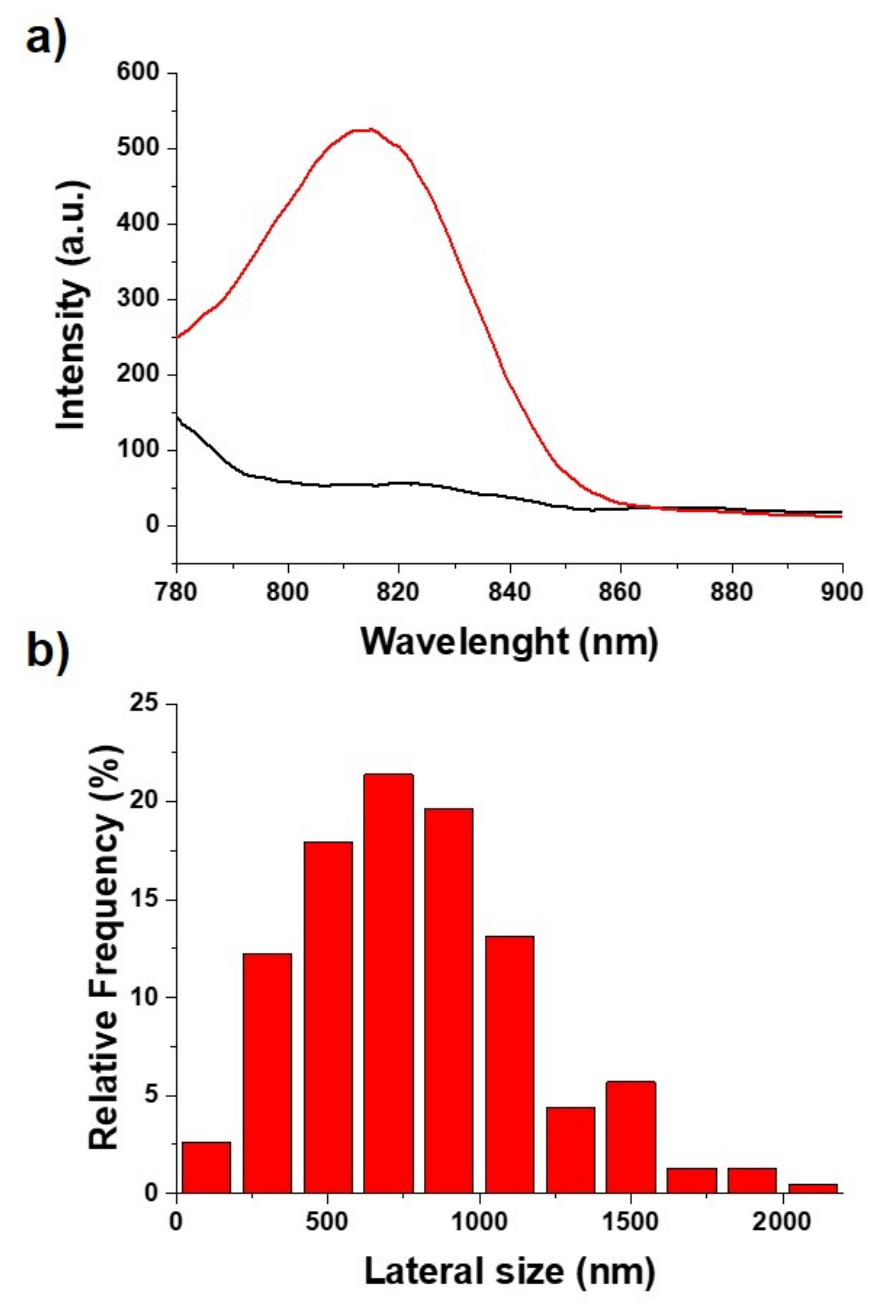

Figure 1: a) Fluorescence of $\mathrm{mfG} / \mathrm{PEG}-\mathrm{FA} / \mathrm{ICG}$ (red line) and control mfG/PEG-FA reaction (black line). Conc. $0.1 \mathrm{mg} / \mathrm{mL}$ of graphene conjugate in NMP. $\lambda_{\mathrm{ex}}: 750 \mathrm{~nm}, \lambda_{\mathrm{em}}: 780-900 \mathrm{~nm}$; b) Histogram of the lateral size distribution of the $\mathrm{mfG} / \mathrm{PEG}-\mathrm{FA} / \mathrm{ICG} / \mathrm{Dox}$

As last step, doxorubicin was introduced onto $\mathrm{mfG}$ through an imine bond. The bond was formed between the last free amines on $\mathrm{mfG}$ and the ketone of Dox to obtain $\mathrm{mfG} / \mathrm{PEG}$ FA/ICG/Dox (mfG-Dox). The characteristics of imine bond is its reversibility in acidic condition, at $\mathrm{pH}<\sim 5.5$. $^{[17]}$ Indeed, the imine bond can be selectively cleaved in tumour microenvironment, where $\mathrm{pH}$ is more acidic than healthy tissues, thanks to an increased therapeutic approach given by the presence of folic acid on graphene. Because of an additional 
amine on Dox structure and its low stability to temperature, the Kaiser test could not be applied to evaluate the loading of drug onto graphene. We evaluated the amount of Dox grafted on $\mathrm{mfG}$ through HPLC analysis by measuring the difference between the initial amount of drug and the quantity of free Dox in solution after the reaction. $\mathrm{mfG}$ were separated from the reaction mixture and steps of dispersion of the material in PBS, centrifugation and removal of the supernatant containing free Dox were repeated until no presence of drug was found in the solution. The total amount of free Dox was measured by HPLC. The quantity of Dox linked to graphene corresponded to $\sim 60 \mu \mathrm{g} / \mathrm{g}(\sim 0.10 \mu \mathrm{mol} / \mathrm{g})$. We hypothesized that the low reaction yield was due to a difficult encounter between the drug and the free amines, due to steric hindrance caused by PEG chain and the other functionalities on the surface of graphene.

The dimensions of the sheets could affect the cells uptake and cytotoxicity. As reported by several studies, sheets with lateral size distribution above $1 \mu \mathrm{m}$ present higher cytotoxicity respect to smaller graphene and graphene oxide sheets. ${ }^{[18-20]}$ Therefore, we evaluate the average lateral size of the particles by statistical analysis using transmission electron microscopy (TEM) images (Figure S2), obtaining a value of $\sim 794 \mathrm{~nm}$, with the presence of few particles smaller than $200 \mathrm{~nm}$ or bigger than $1.5 \mu \mathrm{m}$ (Figure $1 \mathrm{~b}$ ).

Following the synthesis and characterization of the conjugate, we proceed to evaluate its therapeutic potential on cancer cells. In vitro anticancer activity of $\mathrm{mfG}$ nanoconjugate to serve as a drug delivery carrier was evaluated using the water-soluble tetrazolium (WST)-based assay. After $48 \mathrm{~h}$ of incubation at increasing doses ( 5 to $76 \mu \mathrm{g} / \mathrm{mL}$ ), a slight cell mortality was observed for HeLa cells incubated with mfG-Dox and with the control $\mathrm{mfG}$ (corresponding to the functional material without Dox). (Figure S3). The observed cell viability of mfG-Dox and mfG was comparable, with a slight decrease to $\sim 95 \%$ in concentration higher than $50 \mu \mathrm{g} / \mathrm{mL}$. We attributed the failure of the treatment to two main factors: 1) a fast reaggregation of the nanosheets due to low water dispersibility, hindering a high cell uptake, and to a low amount of Dox linked to graphene. To improve the water solubility, we decide to employ bovine serum albumin (BSA) as surfactant. This protein is well known to be a biocompatible dispersant, able to maintain graphene well suspended in water. ${ }^{[21]}$ To increase the quantity of Dox, we decided to exploit the hydrophobicity of graphene surface. Doxorubicin can be adsorbed onto graphene mainly by $\pi$ - $\pi$ stacking interactions, leading to a combined strategy, based on covalently linked and physically adsorbed drug onto graphene surface. To achieve our goal, we sonicated the prepared mfG/PEG-FA/ICG/Dox in a BSA and Dox solution $(1 \mathrm{mg} / \mathrm{mL}$ BSA, ratio $\mathrm{mfG} /$ Dox 1:0.25 w/w. See SI for the method). The final material results to be stable in water and in cell 
culture media for more than $24 \mathrm{~h}$ (mfG/PEG-FA/ICG/Dox at $1 \mathrm{mg} / \mathrm{mL}$ conc.). The total quantity of Dox was evaluated by UV-Vis spectroscopy as difference between the initial amount of Dox and the free drug in solution after absorption, giving a value of $\sim 101 \mu \mathrm{g} / \mathrm{mg}$. With the new conjugate in hand, we first evaluated the drug release efficiency in neutral and acidic conditions ( $\mathrm{pH} 7.4$ and 5.5, respectively), showing a higher drug release at $\mathrm{pH} 5.5$ after $24 \mathrm{~h} \mathrm{(36.7 \% )}$ compared to neutral $\mathrm{pH}$ conditions (14.5\%) (Figure S4). We then evaluated again the in vitro performance. After $24 \mathrm{~h}$ of incubation, a small decrease in the viability of the cells was observed, with a slight difference between the control material and mfG/PEG-FA/ICG/Dox (Figure 2a). However, after $48 \mathrm{~h}$ treatment, Dox conjugated $\mathrm{mfG}$ showed a dose-dependent cytotoxicity to HeLa cells, whereas BSA (vehicle control) and mfG/PEG-FA/ICG (nanocarrier) did not induce any significant difference in cell viability (Figure $2 b$ ). We would like to evidence that $\mathrm{mfG} / \mathrm{PEG}-\mathrm{FA} / \mathrm{ICG} / \mathrm{Dox}$ showed greater toxicity to HeLa cells at all doses as compared to MRC5 normal fibroblasts (Figure S5). As folate receptor (FR) is overexpressed in cancer cells in comparison to normal cells, ${ }^{[22]}$ we hypothesized that such selective anticancer activity was mediated by folate receptor-directed targeting effect. To test this, we subjected HeLa cells to folate starvation and performed an antagonist experiment by pre-incubating these cells with or without an excess of folic acid $(10 \mu \mathrm{M})$. As shown in Figure $2 \mathrm{c}$, the cancer-killing activity of $\mathrm{mfG} / \mathrm{PEG}-\mathrm{FA} / \mathrm{ICG} /$ Dox was retarded by blocking folate receptor. This is likely because the excess of folic acid occupied folate receptors and interfered with the internalization of $\mathrm{mfG}$ conjugates by the cells. Taken together, these results demonstrated that Dox-complexed and folic acid-functionalized $\mathrm{mfG}$ graphene nanosheets could be used as an effective nanocarrier for selective cancer-killing. 
a)

24h treated

-BSA ImfG/PEG-FA/ICG amfG/PEG-FA/ICG/Dox

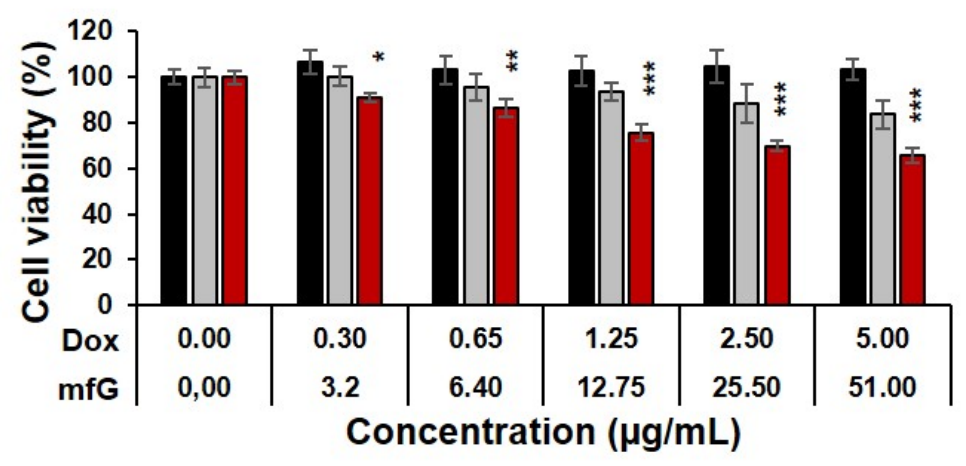

b)

$48 \mathrm{~h}$ treated

-BSA ImfG/PEG-FA/ICG ImfG/PEG-FA/ICG/Dox

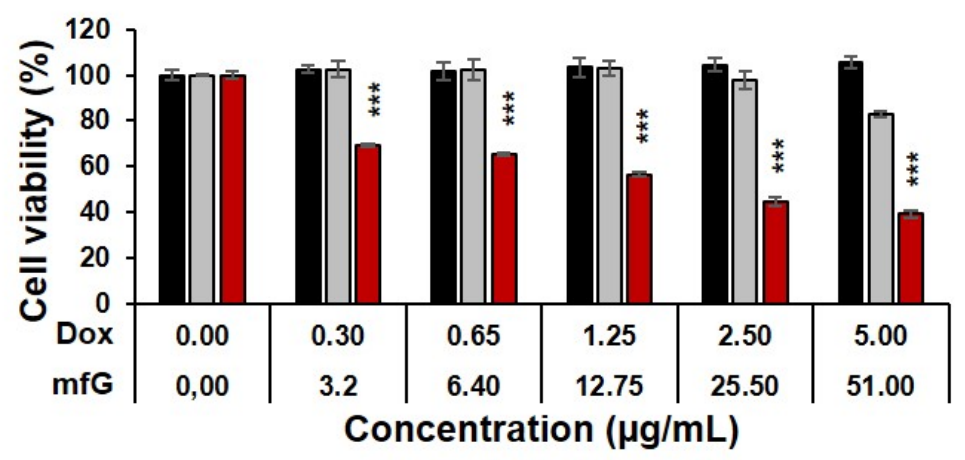

c)

mfG/PEG-FA/ICG/Dox

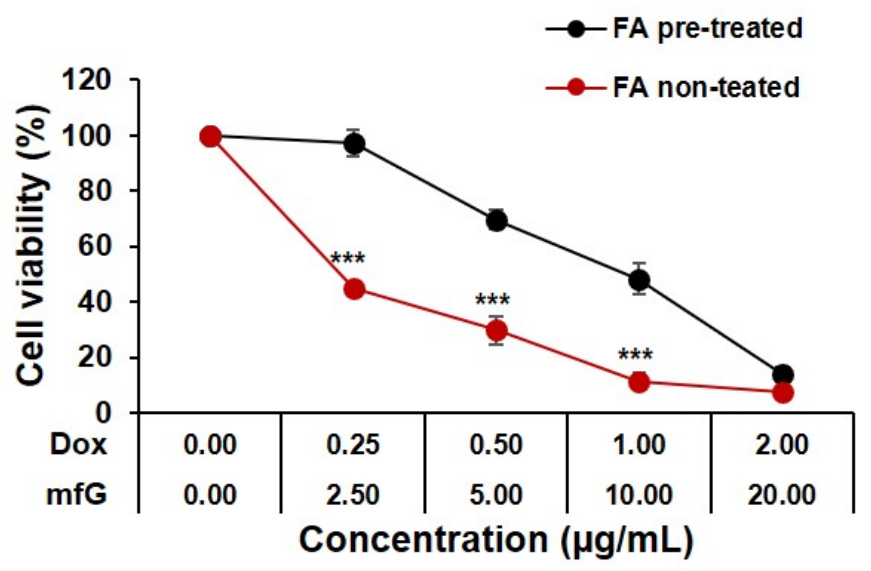

Figure 2: Cells viability experiment on HeLa cells. In $\mathrm{x}$-axis $\mathrm{mfG}$ corresponds to concentration of incubated graphene material, while Dox is the corresponding quantity of drug present on the material. a) Cell viability assay of HeLa cells treated with BSA, mfG/PEG-FA/ICG and mfG/PEG-FA/ICG/Dox for 24 h. Data are expressed as means \pm s.e.m. $(\mathrm{n}=4),{ }^{*} p<0.05,{ }^{* *} p<0.01$, ${ }^{* *} p<0.001$ (Student's t 
test to $0 \mu \mathrm{g} / \mathrm{mL}$ ); b) Cell viability assay of HeLa cells treated with BSA, mfG/PEG-FA/ICG and mfG/PEG-FA/ICG/Dox for 48 h. Data are expressed as means \pm s.e.m. $(\mathrm{n}=4), * * * p<0.001$ (Student's t test to $0 \mu \mathrm{g} / \mathrm{mL}$ ); c) Viability of HeLa cells treated with $\mathrm{mfG} / \mathrm{PEG}-\mathrm{FA} / \mathrm{ICG} /$ Dox for $48 \mathrm{~h}$ with/without pretreatment of free folic acid (FA; $10 \%$ Data are expressed as means \pm s.e.m. $(\mathrm{n}=4),{ }^{* * *} p<0.001$ (Student's $\mathrm{t}$ test comparisons with the equivalent concentration of FA pretreated counterpart).

To visualize the cellular uptake behavior of $\mathrm{mfG}$ nanoconjugates, HeLa cells were incubated with the ICG-labelled $\mathrm{mfG}$ for $4 \mathrm{~h}$ and they were subjected to live cell imaging after washing with PBS. In line with fluorescence spectra results (Figure 1), the fluorescence signals of mfG/PEG-FA/ICG were clearly observed using $800 \mathrm{~nm}$ filter setup (Figure S6a), confirming the successful incorporation of ICG fluorophore onto $\mathrm{mfG}$. The fluorescent image merged with differential interference contrast images (DIC) revealed that $\mathrm{mfG}$ nanoconjugates were accumulated into the cytoplasm, although ICG moieties were partly quenched, as showed by the presence of black aggregates (Figure S6b). To finally confirm the targeting activity and drug release, we observed the release of doxorubicin using confocal fluorescence microscopy. We found that in the complete cell culture medium we can appreciate a release of Dox inside HeLa cells after $4 \mathrm{~h}$ from incubation (Figure S7 and S8). However, a pretreatment with enriched folic acid media (to saturate FA receptors) significantly decreased Dox internalization.

The synergistic effect following the heating of nanomaterials under near-infrared irradiation (NIR) in combination with the enhancing of the activity of Dox at high temperature has been shown to increase the effectiveness of anti-cancer therapy. ${ }^{[23,24]}$ To explore the combination of these two effects exploiting photothermal properties of graphene, we investigated the cell viability of HeLa cells incubated with our drug delivery carrier. HeLa cells were incubated with BSA, ICG and mfG/PEG-FA/ICG/Dox for $4 \mathrm{~h}$ and then have been irradiated for $10 \mathrm{~min}$ using a $785 \mathrm{~nm}$ fiber-coupled continuous-wave NIR laser at $1 \mathrm{~W}\left(\right.$ ca. $\left.8 \mathrm{~W} / \mathrm{cm}^{2}\right)$. The viability of the cells was measured at 0, 24 or $48 \mathrm{~h}$ post-irradiation. As shown in Figure 4, laser irradiation did not alter the viability of the cells treated with BSA and ICG. Interestingly, photoinduced mfG/PEG-FA/ICG/Dox shows no effect on viability immediately after laser irradiation. This behavior could be attributed to the non-sufficient increase in the temperature $\left(\sim 15^{\circ} \mathrm{C} \Delta \mathrm{T}\right.$ from room temperature, Figure S9) due to the low concentration of graphene employed. However, a significant reduction of viability was observed after $24 \mathrm{~h}$ post-irradiation (Figure 3). This behavior can likely be attributed to the synergistic effect of the heat induced by the laser irradiation and a fast Dox release from $\mathrm{mfG}$ by the intracellular acidic environment and laser- 
driven temperature increase, exerting an efficient cell killing effect within the following $24 \mathrm{~h}$ (Figure S10). ${ }^{[24]}$ Despite this first decrease in cells viability, the difference after $48 \mathrm{~h}$ postirradiation is less evident than what observed after $24 \mathrm{~h}$ of incubation (Figure S11a). This is because the cell viability of the different treated cells is converging to the already observed cell viability after $48 \mathrm{~h}$ of incubation (Figure $2 \mathrm{~b}$ and Figure S11b), with a slight decrease of the value for the irradiated cells. Comparing to the similar nanoconjugate based on graphene oxide, laser-irradiated mfG/PEG-FA/ICG/Dox exhibits a better anticancer performance. ${ }^{[23]}$ The observed results are indicating that $\mathrm{mfG} / \mathrm{PEG}-\mathrm{FA} / \mathrm{ICG} / \mathrm{Dox}$ has potential to be used for multimodal synergistic therapy (chemo- and phototherapy) decreasing the activation of the therapy and increasing its effectiveness in cancer-killing activity.

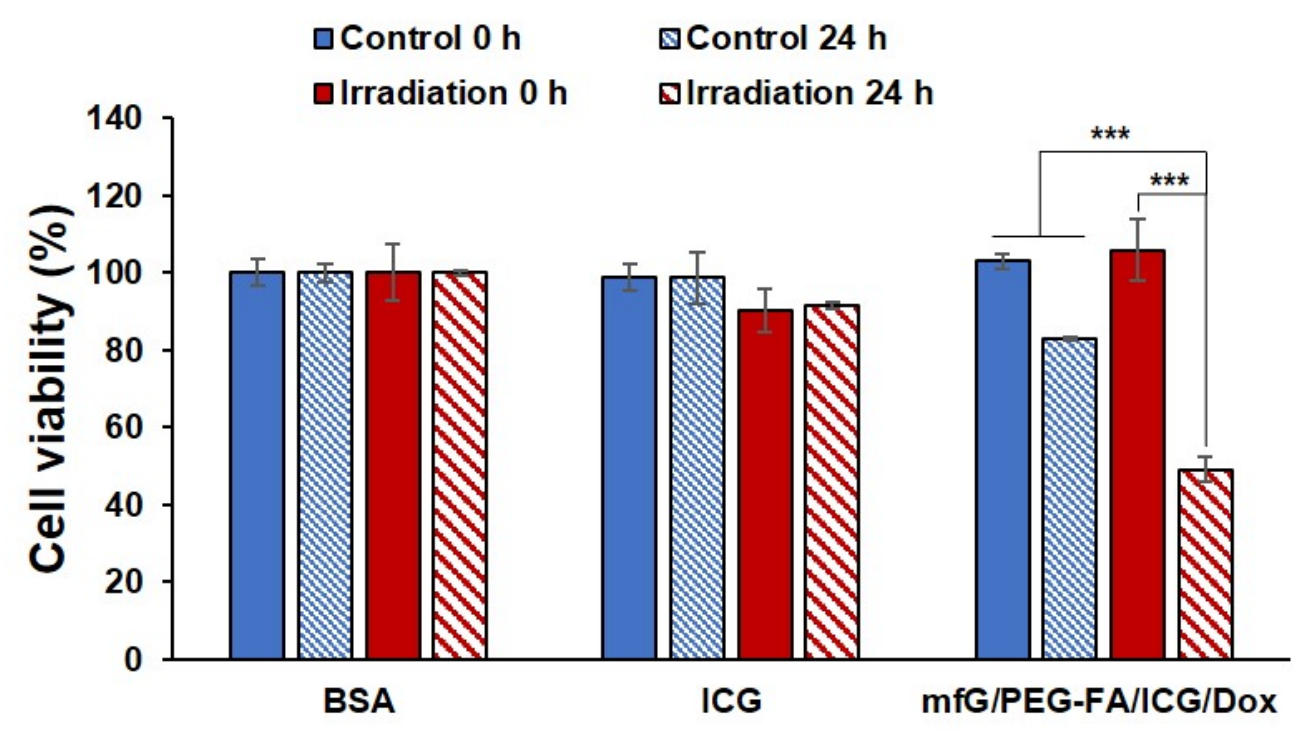

Figure 3: Cell viability of HeLa cells treated with BSA $(20 \mu \mathrm{g} / \mathrm{mL})$, ICG $(0.9 \mu \mathrm{g} / \mathrm{mL})$ and $\mathrm{mfG} / \mathrm{PEG}-$ FA/ICG/Dox $(20 \mu \mathrm{g} / \mathrm{mL})$ with/without $785 \mathrm{~nm}$ laser irradiation for $10 \mathrm{~min}$. The cell viability was tested at 0 and $24 \mathrm{~h}$ after irradiation. The concentrations of BSA and ICG were carefully adjusted to the equivalent amounts loading on the $\mathrm{mfG}$ nanoconjugates. Data are presented as mean \pm s.d. $(\mathrm{n}=3), * * * p$ $<0.001$ (Student's t test).

To assess the biosafety of $\mathrm{mfG}$ nanosheets without modifications (Figure S1), we dispersed the material in BSA solution $(1 \mathrm{mg} / \mathrm{mL})$ and incubated HeLa cells with a wide range of concentrations of $\mathrm{mfG}$ and evaluated cell viability at 24 and $48 \mathrm{~h}$ post treatment. The resulted revealed that $\mathrm{mfG}$ did not affect cell viability even up to the concentration of ca. $150 \mu \mathrm{g} / \mathrm{mL}$, indicating that it is safe for biomedical use (Figure S12). 
Finally, in vivo toxicity evaluation was examined by measuring key blood parameters. Due to low water dispersibility of $\mathrm{mfG}$ (the starting material used for the synthesis of our conjugate), mice were intravenously injected $100 \mu \mathrm{L}$ of the starting $\mathrm{mfG}$ (at $0.5 \mathrm{mg} / \mathrm{mL}, 2.5 \mathrm{mg} \cdot \mathrm{kg}^{-1}$ ) and PBS. Blood test was performed at day 1 and day 7 post injection. The hematological and biochemical parameters did not differ between the groups of $\mathrm{mfG}$ and PBS (Table S1 and S2), demonstrating the lack of acute inflammatory response and underscoring the biosafety of $\mathrm{mfG}$.

To conclude, in this study we designed and synthesized a multifunctional graphene-based platform for cancer therapy applications. We demonstrated the combined chemo- and photothermal properties of the multifunctional graphene as drug delivery carrier, based on the targeting ability of folic acid for HeLa cells, the low in vitro and in vivo toxicity of the starting material and the good cancer killing activity shown by the composite functionalized with doxorubicin. The internalization was confirmed by live microscopy exploiting ICG as fluorophore moiety. As ICG has been also demonstrated to generate reactive oxygen species under irradiation, it could be explored as photodynamic agent. ${ }^{[25,26]}$ The advantages of using graphene as drug carrier were demonstrated in virtue of the synergistic effect of the photo- and chemotherapy after NIR irradiation, making graphene-based material a promising platform for combined cancer therapy applications.

\section{Acknowledgements}

The authors gratefully acknowledge financial support from ANR (ANR-15-GRFL-0001-05) (G-IMMUNOMICS project). This work was partly supported by the Agence Nationale de la Recherche (ANR) through the LabEx project Chemistry of Complex Systems (ANR-10LABX-0026_CSC). M. A. L. is grateful to the Graphene Flagship for receiving a Mobility Grant to Japan. G. A. thanks the financial support from the Spanish MINECO (Unit of Excellence "Maria de Maeztu" MDM-2015-0538), the Generalitat Valenciana (CIDEGENT/2018/001 grant). E. M. thanks the Japan Society for the Promotion of Science KAKENHI Grant-in-Aid for Scientific Research (A) (no. 19H00857) and (B) [no. 16H03834], and the KAKENHI Fund for the Promotion of Joint International Research (no. 16KK0117).

\section{References}

[1] G. Reina, J. M. González-Domínguez, A. Criado, E. Vázquez, A. Bianco, M. Prato, Chem. Soc. Rev. 2017, 46, 4400-4416. 
[2] K. Sharma, in Adv. Pers. Nanotherapeutics, Springer International Publishing, 2017, pp. 57-77.

[3] C. Ménard-Moyon, H. Ali-Boucetta, C. Fabbro, O. Chaloin, K. Kostarelos, A. Bianco, Chem. - A Eur. J. 2015, 21, 14886-14892.

[4] Y. J. Lu, P. Y. Lin, P. H. Huang, C. Y. Kuo, K. T. Shalumon, M. Y. Chen, J. P. Chen, Nanomaterials 2018, 8, 193.

[5] W. Jiang, F. Mo, Y. Lin, X. Wang, L. Xu, F. Fu, J. Mater. Chem. B 2018, 6, 43604367.

[6] H. Wang, Y. Zhao, Y. Wu, Y. lin Hu, K. Nan, G. Nie, H. Chen, Biomaterials 2011, 32, 8281-8290.

[7] Y. Yang, A. M. Asiri, Z. Tang, D. Du, Y. Lin, Mater. Today 2013, 16, 365-373.

[8] B. Fadeel, C. Bussy, S. Merino, E. Vázquez, E. Flahaut, F. Mouchet, L. Evariste, L. Gauthier, A. J. Koivisto, U. Vogel, et al., ACS Nano 2018, 12, 10582-10620.

[9] R. Kurapati, S. P. Mukherjee, C. Martín, G. Bepete, E. Vázquez, A. Pénicaud, B. Fadeel, A. Bianco, Angew. Chemie - Int. Ed. 2018, 57, 11722-11727.

[10] C. Martín, K. Kostarelos, M. Prato, A. Bianco, Chem. Commun. 2019, 55, 5540-5546.

[11] X. Sun, Z. Liu, K. Welsher, J. T. Robinson, A. Goodwin, S. Zaric, H. Dai, Nano Res. 2008, 1, 203-212.

[12] D. A. Jasim, H. Boutin, M. Fairclough, C. Ménard-Moyon, C. Prenant, A. Bianco, K. Kostarelos, Appl. Mater. Today 2016, 4, 24-30.

[13] J. Russier, V. León, M. Orecchioni, E. Hirata, P. Virdis, C. Fozza, F. Sgarrella, G. Cuniberti, M. Prato, E. Vázquez, et al., Angew. Chemie - Int. Ed. 2017, 56, 3014-3019.

[14] M. A. Lucherelli, J. Raya, K. F. Edelthalhammer, F. Hauke, A. Hirsch, G. Abellán, A. Bianco, Chem. - A Eur. J. 2019, chem.201903165.

[15] V. K. Sarin, S. B. H. Kent, J. P. Tam, R. B. Merrifield, Anal. Biochem. 1981, 117, 147157.

[16] H. S. S. Ramakrishna Matte, K. S. Subrahmanyam, K. Venkata Rao, S. J. George, C. N. R. Rao, Chem. Phys. Lett. 2011, 506, 260-264. 
[17] S. Xu, Y. Luo, R. Haag, Macromol. Biosci. 2007, 7, 968-974.

[18] G. Qu, X. Wang, Q. Liu, R. Liu, N. Yin, J. Ma, L. Chen, J. He, S. Liu, G. Jiang, J. Environ. Sci. (China) 2013, 25, 873-881.

[19] K. P. Wen, Y. C. Chen, C. H. Chuang, H. Y. Chang, C. Y. Lee, N. H. Tai, J. Appl. Toxicol. 2015, 35, 1211-1218.

[20] S. Syama, W. Paul, A. Sabareeswaran, P. V. Mohanan, Biomaterials 2017, 131, 121130.

[21] J. I. Paredes, S. Villar-Rodil, Nanoscale 2016, 8, 15389-15413.

[22] J. A. Ledermann, S. Canevari, T. Thigpen, Ann. Oncol. 2015, 26, 2034-2043.

[23] W. Zhang, Z. Guo, D. Huang, Z. Liu, X. Guo, H. Zhong, Biomaterials 2011, 32, 85558561.

[24] H. Park, J. Yang, J. Lee, S. Haam, I.-H. Choi, K.-H. Yoo, ACS Nano 2009, 3, 29192926.

[25] K. Urbanska, B. Romanowska-Dixon, Z. Matuszak, J. Oszajca, P. Nowak-Sliwinska, G. Stochel, Acta Biochim. Pol. 2002, 49, 387-391.

[26] Y. Yu, X. Yang, M. Liu, M. Nishikawa, T. Tei, E. Miyako, ACS Appl. Bio Mater. 2019, 2, 3693-3705. 


\section{Graphical Abstract}

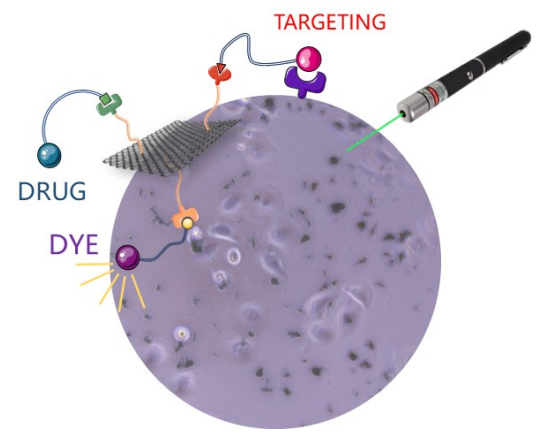

A multifunctional drug delivery platform based on graphene, functionalized with indocyanine green, folic acid and doxorubicin was designed and prepared, leading to an efficient combined chemo- and photothermal effect in cancer therapy. 